\title{
Susceptibilidade a nematóides em ovelhas Santa Inês, Bergamácia e Texel no Noroeste do Paraná
}

\section{Susceptibility to nematodes of Santa Inês, Bergamácia and Texel ewes on northwest of Paraná}

\author{
Alexandre Agostinho Mexia ${ }^{*}$; Francisco de Assis Fonseca de Macedo $^{2}$; Carlos \\ Antonio Lopes de Oliveira ${ }^{2}$; Marilice Zundt ${ }^{3}$; Sandra Mari Yamamoto ${ }^{4}$; Graziela \\ Aparecida Santello ${ }^{5}$; Renata Del Carratore Carneiro ${ }^{6}$; Aya Sasa ${ }^{7}$
}

\begin{abstract}
Resumo
Com o objetivo de verificar a susceptibilidade de ovelhas à infecção por nematóides gastrointestinais foi realizado experimento no Centro de Pesquisa do Arenito (UEM), no município de Cidade Gaúcha, noroeste do Paraná. Foram utilizadas 94 ovelhas Santa Inês, 14 Bergamácia e 25 Texel. Os rebanhos permaneceram em pastagem de Tanzânia (Panicum maximum cv. Jaq) no período diurno, sendo recolhidos em instalações cobertas, com piso ripado suspenso, no período noturno, onde foi fornecido resíduo de fécula de mandioca. As ovelhas foram monitoradas mensal e individualmente quanto à carga endoparasitária, por meio de contagem de ovos por grama de fezes (OPG), e realizada cultura de larvas para identificação das espécies de endoparasitos. As ovelhas Santa Inês apresentaram menor $(P \leq 0,05)$ susceptibilidade comparada às raças Texel e Bergamácia, sendo as médias de OPG de 838,58; 1240,31 e 1821,33 , respectivamente. A maior porcentagem de endoparasitos correspondeu à espécie Haemonchus contortus, demonstrando que este parasito foi o principal responsável pelos valores encontrados de OPG, seguido por Trichostrongylus colubriformis. A contagem de OPG das ovelhas Santa Inês apresentou comportamento cúbico ao longo do ano de observação, atingindo pico máximo em outubro, mês em que se concentraram as parições. As ovelhas Texel e Bergamácia também apresentaram comportamento cúbico ao decorrer do ano, contudo, foram observadas maiores contagens de OPG nos meses de maio, junho e julho. As ovelhas Santa Inês foram menos susceptíveis à infecção por endoparasitos.
\end{abstract}

Palavras-chave: Cultura de larvas, endoparasitos, Haemonchus contortus, OPG

\begin{abstract}
Aiming to measure the resistance of ewes against gastrintestinal nematodes, an experiment was conducted at Centro de Pesquisa do Arenito, of the Universidade Estadual de Maringá, located in the city of Cidade Gaúcha, northwest of Paraná. Ninety- four Santa Inês, 14 Bergamácia and 25 Texel ewes were used. The
\end{abstract}

\footnotetext{
1 Prof. do Dept ${ }^{\mathrm{o}}$ de Zootecnia, Universidade do Estado de Mato Grosso, UNEMAT, Pontes e Lacerda, MT. E-mail: alexandre@ unemat.br

2 Prof. do Dept ${ }^{\circ}$ de Zootecnia, Universidade Estadual de Maringá, UEM, Maringá, PR. E-mail: fafmacedo@uem.br; caloliveira@ uem.br

3 Prof ${ }^{\mathrm{a}}$. do Dept ${ }^{\mathrm{o}}$ de Zootecnia, Universidade do Oeste Paulista, UNOESTE, Presidente Prudente, SP. E-mail: mari@unoeste.br

4 Prof ${ }^{\text {a }}$ do Colegiado de Zootecnia, Universidade Federal do Vale do São Francisco, UNIVASF. Petrolina, PE. E-mail: syamamoto@ univasf.br

5 Prof $^{a}$. do Dept ${ }^{\circ}$ de Zootecnia, Universidade Federal do Amazonas, Paritins, AM. E-mail: grasantello@hotmail.com

6 Prof ${ }^{\mathrm{a}}$. do Dept ${ }^{\mathrm{o}}$ de Agronomia, Universidade do Estado de Mato Grosso, UNEMAT, Nova Xavantina, MT. E-mail: carneirodel@, ig.com.br

7 Prof $^{\mathrm{a}}$. do Dept ${ }^{\mathrm{o}}$ de Zootecnia, Universidade Estadual de Mato Grosso do Sul, UEMS, Aquidauana, MS. E-mail: aya@uems.br

* Autor para correspondência
}

Recebido para publicação 19/08/2011 Aprovado em 19/10/2011 
flock remained on Tanzânia (Panicum maximum cv. Jaq) pasture during the day, being withdrawn indoors, with suspended battened floor, during the night, when they were provided cassava bagasse. The ewes were monitored monthly and individually regarding the amount of endoparasites, through egg counting per gram of feces (EPG), and worms culture for identification of the species of those endoparasites. The Santa Inês ewes presented had lower $(\mathrm{P} \leq 0,05)$ susceptibility comparing to the breeds Texel and Bergamácia, being the averages of EPG 838,58; 1240,31 and 1821,33 respectively. The highest percentage of endoparasites corresponded to the Haemonchus contortus, showing that this was the main type of endoparasites responsible for the EPG levels, followed by Trichostrongylus colubriformis. The counting of EPG of Santa Inês ewes showed a cubical behaviour over the year of observation, reaching the higher level in October, month in which the giving births happened. The Texel and Bergamácia ewes also showed cubical behaviour during the year, however, the higher EPG levels happened in the months of May, June and July. The Santa Inês ewes were the least prone to infection by endoparasites.

Key words: Endoparasites, EPG, Haemonchus contortus, worms culture

\section{Introdução}

Embora a bovinocultura seja a principal atividade pecuária no noroeste do Paraná, nos últimos anos a ovinocultura vem se desenvolvendo consideravelmente nesta região, apresentando-se como mais uma opção para os empresários rurais.

Em relação à criação de ovinos, as verminoses representam o maior e mais grave problema sanitário concernente à ovinocultura, e podem inviabilizar economicamente a criação (BUZZULINI et al., 2007). Os animais criados a pasto estão permanentemente expostos à contaminação parasitária, pois, do total da carga parasitária ovina, estima-se que mais de $95 \%$ encontra-se nas pastagens (BOWMAN; LYNN; EBEHARD, 2003).

O conhecimento real de tal prejuízo ainda é desconhecido (MOLENTO et al., 2004), porém as principais características da infecção gastrintestinal por helmintos são a severa depressão da capacidade digestiva e de absorção na mucosa do local da infecção (MATTOS et al., 2005), podendo reduzir o ganho de peso e a conversão alimentar, além de comprometer o desempenho reprodutivo e o sistema imunológico (COSTA et al., 2004).

Amarante (2001) confirma que a principal espécie endoparasita que acomete ovinos no Brasil é a Haemonchus contortus, sendo encontrados em $75 \%$ a $100 \%$ dos exames de contagem de ovos por grama de fezes (MORTENSEN et al., 2003).
Devido ao hábito hematófago deste parasito abomasal, animais com altos níveis parasitários poderão perder grande quantidade de sangue, desenvolvendo um quadro de anemia grave, em um curto período de tempo. As respostas imunológicas contra a reinfecção se desenvolvem de maneira lenta e incompleta, deixando os rebanhos sujeitos à reincidência das formas clínicas e subclínicas dessa parasitose (VIEIRA, 2010).

SegundoAmarante (2001), em seguida, em ordem de importância, aparece a espécie Trichostrongylus colubriformis, parasita do intestino delgado presente em praticamente todas as criações de ovinos. Estes vermes lesam a mucosa intestinal, provocando exsudação de proteínas séricas para a luz intestinal.

A principal manifestação clínica da ação do Haemonchus contortus caracteriza-se por anemia e hipoproteinemia, levando ao aparecimento de mucosas pálidas, edema submandibular e baixo valor de hematócrito ao hemograma (LOURENÇO, 2006).

De uma forma geral, os animais, até a puberdade, apresentam grande susceptibilidade à verminose. A resistência aumenta na idade adulta, porém existem determinadas épocas e condições fisiológicas em que o animal encontra-se mais susceptível (MILLER; HOROBOV, 2006). Diante disso, são necessárias estratégias adequadas de controle que devem basear-se, principalmente, na época do ano e estado fisiológico do animal (BAGNOLA JÚNIOR; 
AMARANTE; MEYER, 1996).

Erros inerentes ao uso indevido de certas raças são constantes em todo o Brasil, em várias espécies animais. Uma das alternativas ao menor uso de antihelmínticos é a criação de ovinos que apresentam menor suscetibilidade aos parasitas.

Objetivou-se neste trabalho verificar o comportamento de ovelhas de diferentes grupos genéticos à infecção por nematóides gastrintestinais, através de monitoramento da contagem de OPG.

\section{Material e Métodos}

O experimento foi realizado no Centro de Pesquisa do Arenito (UEM), em Cidade Gaúcha, noroeste do Paraná. Esta região situa-se a 23025 'de latitude Sul, 510 55' de longitude Oeste e 554,9 m de altitude. O clima predominante, segundo Corrêa (1996), é classificado como subtropical úmido mesotérmico com verões quentes, geadas pouco freqüentes, com concentração de chuvas nos meses de verão e o solo classificado como Podzólico vermelho-amarelo de textura média (SECRETARIA DO ESTADO DO PARANÁ, 1985).

Foram utilizadas 94 ovelhas Santa Inês, 14 ovelhas Bergamácia e 25 ovelhas Texel. A estação de monta teve início em maio de 2001, com 45 dias de duração.

Durante o experimento, os rebanhos permaneceram em pastagem de capim Tanzânia (Panicum maximum cv. Jaq) no período diurno, sendo recolhidos em instalações cobertas, com piso ripado e suspenso, no período noturno, para proteção contra predadores e menor disposição à infecção por helmintos parasitas. Após todas as ovelhas serem recolhidas às instalações era fornecido, à vontade, resíduo de fécula de mandioca. As ovelhas tiveram acesso à mistura mineral, em cochos separados.

Aradiaçãosolarfoimedidaatravésdoactemógrafo e a precipitação pluviométrica por pluviômetro (Ville Paris). Os dados bioclimatológicos coletados em Paranavaí foram fornecidos pelo Centro de Agrometeorologia - área de ecofisiologia, IAPAR Londrina, sendo apresentados na Figura 1.

Na primeira semana após a parição, as ovelhas permaneceram confinadas com seus cordeiros durante 24 horas. Posteriormente, até a terceira semana, somente as ovelhas tiveram acesso à pastagem 4 horas/dia, no período vespertino. Da quarta semana até o desmame (60 dias), as ovelhas permaneceram durante o dia em pastagem, sendo recolhidas no final da tarde.

Figura 1. Precipitação pluviométrica $\left(\mathrm{mm}^{3}\right)$ e radiação solar (cal $/ \mathrm{cm}^{2} /$ dia), mensal de fevereiro a janeiro.

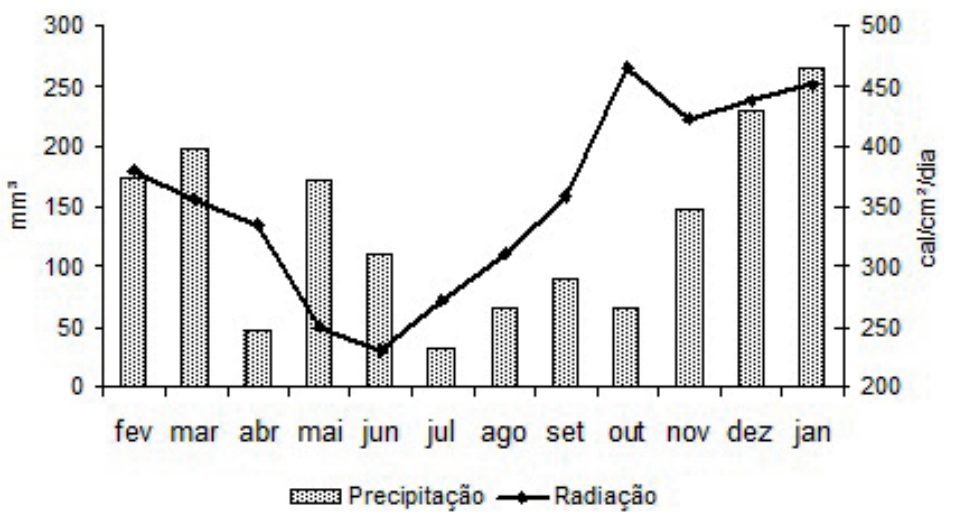

Fonte: Elaboração dos autores. 
As ovelhas foram monitoradas mensal e individualmente quanto à carga endoparasitária, por meio de contagem de ovos por grama de fezes (OPG) segundo Gordon e Whitlock (1939). Sempre que a contagem excedeu a 500 o animal foi everminado com moxidectin $\left(\right.$ Cydectin $^{\circledR}$, FordDodge) na dosagem de $200 \mu \mathrm{g} / \mathrm{kg}$ de PV.

Após a aplicação de anti-helmíntico, os animais permaneciam 18 a 24 horas nas instalações com piso ripado e suspenso, quando então eram conduzidos a um piquete não freqüentado por ovinos há, pelo menos, 45 dias.

Ainda, realizou-se cultura de larvas para identificação das principais espécies de nematóides, mediante técnica de coprocultura de Ueno e Gonçalves (1994).

Os dados de OPG foram analisados pela metodologia de modelos lineares generalizados (NELDER; WEDDERBURN, 1972). Foi assumida a distribuição binomial negativa e a função de ligação a logarítmica que torna $\eta$ uma função linear dos parâmetros. As informações de OPG dos animais foram analisadas empregandose o software ASREML (GILMOUR et al., 2000).

\section{Resultados e Discussão}

Os resultados referentes à contagem de OPG das ovelhas Santa Inês, Texel e Bergamácia ao longo do ano estão expressos na Tabela 1. Podemos observar que a raça Santa Inês apresentou menor susceptibilidade $(\mathrm{P} \leq 0,05)$ ao endoparasitismo que as raças Texel e Bergamácia, sendo que estas duas últimas não diferiram entre si $(P>0,05)$. Ainda, em relação aos animais Santa Inês, foi observado que $13,40 \%$ apresentavam valores de OPG acima de 500 durante o período experimental. Para os animais Texel e Bergamácia, 19,84 e 21,13\% dos animais, respectivamente, necessitaram de tratamentos individuais.
Tabela 1. Contagem média de ovos por grama de fezes (OPG) nos exames coprológicos das ovelhas nos diferentes grupos genéticos, de fevereiro 2001 a janeiro 2002, no noroeste do Paraná.

\begin{tabular}{lc}
\hline Grupo genético & OPG \\
\hline Santa Inês & $838.58 \pm 76.14 \mathrm{a}$ \\
Texel & $1240.31 \pm 228.65 \mathrm{~b}$ \\
Bergamácia & $1821.33 \pm 442.07 \mathrm{~b}$ \\
\hline
\end{tabular}

Médias seguidas de letras diferentes indicam valores diferentes $(\mathrm{P} \leq 0,05)$ pelo teste de Tukey.

Ao caracterizar ovelhas e borregas quanto infecção natural por parasitas gastrointestinais Bueno et al. (2002) observaram que animais da raça Suffolk apresentaram valores de OPG superiores a todas raças testadas. Ovinos Poll Dorset e Ile de France apresentaram valores intermediários e Santa Inês, tanto borregas quanto adultas, inferiores (1430; 947; 977; 615; 342 respectivamente).

A capacidade do animal de apresentar baixa carga parasitária é denominada de resistência ao endoparasitismo. Um dos mecanismos que explica essa resistência é a resposta imune que envolve o reconhecimento do antígeno do nematóide, seguido da estimulação do sistema imunológico para gerar a resposta apropriada da mucosa do trato digestivo e, finalmente, a liberação de produtos que promovam a eliminação do parasita do trato digestivo (DOUCH, 1990). Os mecanismos mais importantes de resistência são a produção de Imunoglobulina do tipo A, que regula a fecundidade do endoparasito, e a geração de uma resposta hipersensível imediata, que regula a carga de endoparasitos (STEAR et al., 1997).

McManus et al. (2009) ao trabalharem com valores de OPG em animais Santa Inês, Bergamácia, Ile de France, Morada Nova, cruza IlexSI e TexelxSI, concluíram com base nos resultados que estratégias de seleção genética de ovinos destinadas a reduzir infecções gastrointestinal possívelmente selecionam animais mais resistentes. Amarante et al. (1999) por vossa vez, ao compararem ovelhas 
das raças Florida Nativa, Rambouillet e ovelhas mestiças Florida Nativa x Rambouillet, observaram que as ovelhas da raça Rambouillet se mostraram mais suscetíveis ao parasitismo, apresentando maior contagem de OPG.

Avaliando o comportamento parasitológico de fêmeas ovinas através do valor do hematócrito e contagem de ovos por grama de fezes (OPG) na identificação de animais severamente parasitados, Lourenço (2006) observou que fêmeas ovinas das raças Santa Inês e Ile de France apresentaram valores médios de hematócrito superiores às Texel, evidenciando a influência do grupo racial sobre o hematócrito.

A contagem de OPG das ovelhas Santa Inês apresentou comportamento cúbico ao longo do ano de observação [Y= exp $\left(7,573-0,8182 \mathrm{M}+0,1612 \mathrm{M}^{2}\right.$ $\left.0,008482 \mathrm{M}^{3}\right)$ ]. No mês de maio, as ovelhas Santa Inês apresentaram valor médio de OPG próximo a 564, iniciando-se aumento nos valores a partir deste mês, alcançando valores superiores a $1100 \mathrm{em}$ outubro, época em que se concentraram as parições deste grupo racial (Figura 2).

$\begin{array}{ccccr}\text { As } & \text { ovelhas } & \text { do } & \text { grupo genético } & \text { Texel } \\ {[\mathrm{Y}=} & \exp & & \left(2,572+2,629 \mathrm{M}-0,3913 \mathrm{M}^{2}\right.\end{array}$

$\left.\left.+0,01675 \mathrm{M}^{3}\right)\right]$ e Bergamácia $\quad[\mathrm{Y}=\exp$ $\left.\left(3,591+2,139 \mathrm{M}-0,3079 \mathrm{M}^{2}+0,01277 \mathrm{M}^{3}\right)\right]$, também apresentaram comportamento cúbico no decorrer do ano, porém com maior contagem de OPG nos meses de menor radiação solar. Os valores médios foram 3066 e 3585 OPG, para o grupo Texel e Bergamácia, respectivamente, no mês de maio, coincidindo com o período periparto destas ovelhas (Figura 2).

O aumento de OPG a partir do parto e sua diminuição ao longo dos meses, caracterizaram a ocorrência do fenômeno periparto em todos os grupos raciais estudados. Otto et al. (1994) concluíram que durante o periparto (final da gestação e início da lactação), as ovelhas apresentam maior eliminação de ovos de parasitas por grama de fezes, e com a interrupção da lactação, as ovelhas tornam-se mais resistentes à verminose.

Ciarlini et al. (2002) verificaram valores médios de OPG de 1300 e 5375 em ovelhas Suffolk mantidas em pastagem no pré-parto e lactação, respectivamente. Fernandes et al. (2004) observaram valores acima de 3000 OPG em ovelhas na fase de periparto cruzadas Ile de France, também mantidas em sistema de pastejo.

Figura 2. Contagem média de ovos por grama de fezes (OPG) nos exames coprológicos das ovelhas Santa Inês, Texel e Bergamácia, de fevereiro de 2001 a janeiro de 2002.

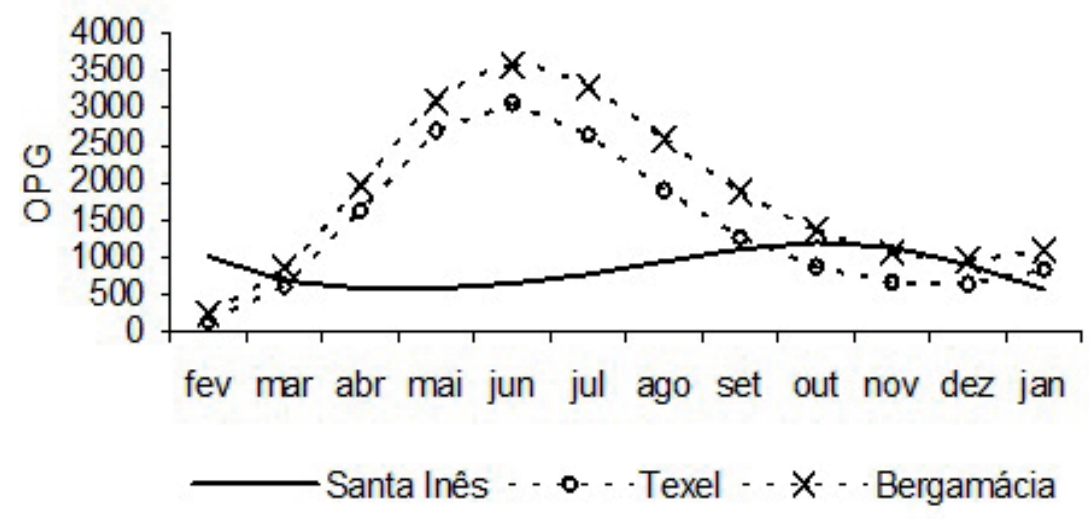

Fonte: Elaboração dos autores. 
Em geral, o periparto (terço final da gestação e início da lactação) é o período em que as ovelhas se tornam mais susceptíveis às infecções por nematódeos gastrintestinais. Isso pode ocorrer devido à queda de imunidade (JANSEN, 1987; GREER, 2008), e também pelo aumento da exigência nutricional nessa fase (NRC, 1985; HOUDIJK, 2008).

Amarante et al. (2004) ao avaliarem a resistência contra infecções por nematóides em animais das raças Santa Inês, Ile de France e Suffolk por 9 meses apartir do inverno de 2000, observaram dois picos na contagem de ovos para os animais Santa Inês, um sendo em janeiro e o outro em maio. Ainda notaram que os valores médios de $\mathrm{H}$. contortus eram muito mais baixas em ovelhas Santa Ines quando comparadas a Suffolk e Ile de France, apesar de estas duas últimas receberem tratamentos anti-helmíntico com maior frequência.

No presente estudo, podemos observar (Figura 3) maior porcentagem de larvas da espécie Haemonchus contortus em relação à espécie Trichostrongylus columbriformis, demonstrando que esta foi a principal espécie responsável pelos valores encontrados de OPG.

A maior concentração da espécie Haemonchus. contortus ocorreu no mês de outubro e dezembro, representando 98\% e 97\%, respectivamente. Em junho, $76 \%$ das larvas recuperadas foi correspondente a espécie Trichostrongylus columbriformes.

Outros autores (FERNANDES et al., 2004; ROCHA; AMARANTE; BRICARELLO, 2005; PEGORARO et al., 2008) já constataram a prevalência desta espécie de parasita. Ainda, outros autores constataram predominância do gênero Haemonchus nas coproculturas realizadas após a administração de anti-helmínticos, comprovando resistência (MELO et al., 2003; THOMAZSOCCOL et al., 2004).

Neste sentido, destaca-se a pesquisa realizada por Amarante et al. (1996), que ao trabalharem com ovelhas na região de Botucatu/SP, verificaram, em ordem decrescente de contagem de OPG, os seguintes gêneros: Haemonchus, Trichostrongylus, Cooperia e Oesophagostomum, sendo a contagem mais elevada em agosto, apresentando 2319,3; 222,2; 420,6 e 0,0, respectivamente.

Martin Nieto et al. (2003) observaram em Cidade Gaúcha, PR, que a maior porcentagem de parasitas correspondeu ao $H$. contortus, principalmente durante julho a outubro de 1998 e de janeiro a abril de 1999, entretanto, não observou diferença entre os grupos genéticos empregados (fêmeas Corriedale com machos Bergamácia e Hampshire Down).

Figura 3. Percentagem de larvas recuperadas das culturas coprológicas de fevereiro de 2001 a janeiro de 2002.

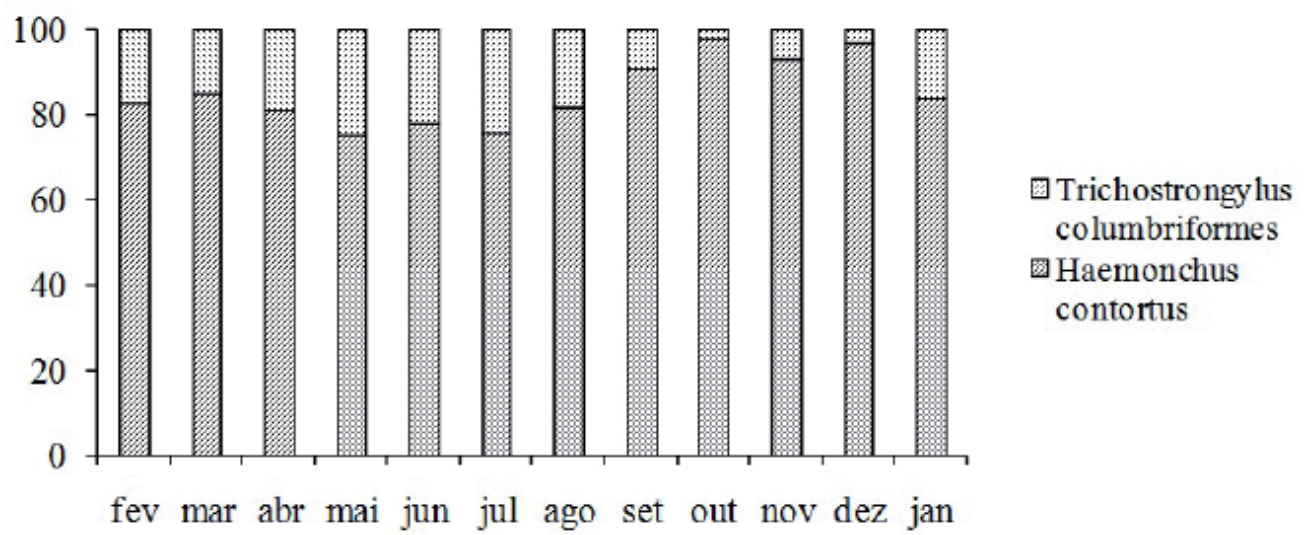

Fonte: Elaboração dos autores. 


\section{Conclusões}

As ovelhas Santa Inês foram menos susceptíveis comparadas às raças Texel e Bergamácia à infecção por endoparasitos quando manejadas a campo, podendo ser recomendada como formação de rebanho materno, quando se leva em consideração, principalmente, a resistência.

Os rebanhos de Texel e Bergamácia merecem maior atenção ao manejo sanitário, principalmente próximo aos meses de maio a julho, caso a cobertura tenha sido realizada próxima ao início do ano.

\section{Referências}

AMARANTE, A. F. T.; CRAIG, T. M.; RAMSEY, W. S.; EL-SAYED, N. M.; DESOUKI, A. Y.; BAZER, F. W. Comparison of naturally acquired parasite burdens among Florida Native, Rambouillet and crossbreed ewes. Veterinary Parasitology, Uberaba, v. 85, n. 1, p. 61-69, 1999.

AMARANTE, A. F. T.; BRICARELLO, P. A.; ROCHA, R. A.; GENNARI, S. M. Resistance of Santa Ines, Suffolk and Ile de France sheep to naturally acquired gastrointestinal nematode infections. Veterinary Parasitology, Uberaba, v. 120, n. 1-2, p. 91-106, 2004.

AMARANTE, A. F. T.; PADOVANI, C. R.; BARBOSA, M. A. Contaminação da pastagem por larvas infectantes de nematódeos gastrintestinais parasitas de bovinos e ovinos em Botucatu-SP. Revista Brasileira de Parasitologia Veterinária, São Paulo, v. 5, n. 2, p. 65-73, 1996.

AMARANTE, A. F. T. Controle de endoparasitoses dos ovinos. In: A PRODUÇÃO ANIMAL NA VISÃO DOS BRASILEIROS, 2001, Piracicaba. Palestras... Piracicaba: FEALQ, 2001. p. 461-473.

BAGNOLA JUNIOR, J.; AMARANTE, A. F. T.; MEYER, L. F. Verminose em eqüinos: exames parasitológicos, contaminação da pastagem e pastejo alternado com ovinos. Veterinária e Zootecnia, São Paulo, v. 8, p. 47-57, 1996.

BOWMAN, D. D.; LYNN, R. C.; EBEHARD, M. L. Georgi's parasitology for veterinarians. $8^{\text {th }}$ ed. St. Louis: Saunders, 2003. $422 \mathrm{p}$.

BUENO, M. S.; CUNHA, E. A.; VERÍSSIMO, C. J.; SANTOS, L. E.; LARA, M. A. C.; OLIVEIRA, S. M.; SPÓSITO FILHA, E.; REBOUÇAS, M. M. Infección por nematodos en razas de ovejas cárnicas criadas intensivamente en la región del sudeste del Brasil. Archivos de Zootecnia, Córdoba, v. 51, n. 193, p. 271278, 2002.

BUZZULINI, C.; SILVA SOBRINHO, A. G.; COSTA, A. J.; SANTOS, T. R.; BORGES, F. A.; SOARES, V. E. Eficácia anti-helmíntica comparativa da associação albendazole, levamisole e ivermectina à moxidectina em ovinos. Pesquisa Agropecuária Brasileira, Brasília, v. 42, n. 6, p. 891-895, 2007.

CIARLINI, P. C.; CIARLINI, L. D. R. P.; ALENCAR, N. X.; KOHAYAGAWA, A.; RODRIGUES, C. F. C. Metabolismo oxidativo de neutrófilos em ovelhas naturalmente infectadas por nematódeos gastrintestinais e correlação entre nível sérico de cortisol e carga parasitária. Arquivo Brasileiro de Medicina Veterinária e Zootecnia, Belo Horizonte, v. 54, n. 3, p. 242-247, 2002.

CORRÊA, A. R. Forrageiras: aptidão climática do Estado do Paraná. In: MONTEIRO A. L. G.; MORAES, A.; CORRÊA, E. A. S.; OLIVEIRA, J. C.; SÁ, J. P. G.; ALVES, S. J.; POSTIGLIONI, S. R.; CECATO, U. Forragicultura do Paraná. Londrina, 1996, p. 75-92.

COSTA, A. J.; OlIVEIRA, G. P.; ARANTE, P. T.; BORGES, F. A.; MENDONCA, R. P.; SANTANA, L. F.; SAKAMOTO, C. A. M. Avaliação comparativa da ação antihelmíntica e do desenvolvimento ponderal de bezerros tratados com diferentes avermectinas de longa ação. A Hora Veterinária, v. 24, n. 139, p. 31-34, 2004.

DOUCH, P. G. C. The sheep,s imune response to nematode parasites and prospects for its exploitation. In: PROCEEDINGS OF THE NEW ZEALAND SOCIETY OF ANIMAL PRODUCTION, 1990. Proceedings... New Zealand, 1990. v. 50, p. 229-236.

FERNANDES, L. H.; SENO, M. C. Z.; AMARANTE, A. F. T.; SOUZA, H.; BELLUZZO, C. E. C. Efeito do pastejo rotacionado e alternado com bovinos adultos no controle da verminose em ovelhas. Arquivos Brasileiro de Medicina Veterinária e Zootecnia, Belo Horizonte, v. 56, n. 6, p. 733-740, 2004.

GILMOUR, A. R.; CULLIS, A. R.; WELHAM, S. J.; GOGEL, B. J.; THOMPSON, R. ASREML, reference manual. Austrália: NSW Agriculture, Orange, 2000. 218 p.

GORDON, H.; WHITLOCK. H. V. A new tecnique for counting nematode eggs in sheep faeces. Journal Council Science \& Industry Research, n. 29, p. 60-67, 1939.

GREER, A. W. Trade-offs and bene fits: implications of promoting a strong immunity to gastrointestinal parasites in sheep. Parasite Immunology, Mansoura, v. 30, n. 2, p. 123-132, 2008. 
HOUDIJK, J. G. M. Influence of periparturient nutritional demand on resistance to parasites in livestock. Parasite Immunology, Mansoura, v. 30, n. 2, p. 113-121, 2008.

JANSEN, J. The peri-parturient rise in sheep. Feacal worm egg coutns in normal and late lambing ewes. The Veterinary Quarterly, Dordrecht, v. 9, n. 2, p. 97-102, 1987.

LOURENÇO, F. J. Utilização de diferentes métodos para detecção do comportamento endoparasitário em fêmeas ovinas de diferentes grupos raciais. 2006. Dissertação (Mestrado em Zootecnia) - Universidade Estadual de Maringá, Maringá.

MARTIN NIETO, L.; MARTINS, E. N.; MACEDO, F. A. F.; ZUNDT, M. Observações epidemiológicas de helmintos gastrintestinais em ovelhas mestiças manejadas em pastagens com diferentes hábitos de crescimento. Ciência Animal Brasileira, Goiânia, v. 4, n. 1, p. 45-51, 2003.

MATTOS, M. J. T.; OLIVEIRA, C. M. B.; LUSTOSA, A.; LACERDA, L. A.; TERRA, S. Influência do parasitismo por nematódeos sobre o perfil hematológico de caprinos. Arquivo Brasileiro de Medicina Veterinária e Zootecnia, Belo Horizonte, v. 57, n. 1, p. 133-135, 2005.

MCMANUS, C.; LOUVANDINI, H.; PAIVA, S. R.; OLIVEIRA, A. A.; AZEVEDO, H. C.; MELO, C. B. Genetic factors of sheep affecting gastrointestinal parasite infections in the Distrito Federal, Brazil. Veterinary Parasitology, Uberaba, v. 166, n. 3/4, p. 308-313, 2009.

MELO, A. C. F. L.; REIS, I. F.; BEVILAQUA, C. M. L.; VIEIRA, L. S.; ECHEVARRIA, F. A. M.; MELO, L. M. Nematódeos resistentes a anti-helmíntico em rebanhos de ovinos e caprinos do estado do Ceará, Brasil. Ciência Rural, Santa Maria, v. 33, n. 2, p. 339-344, 2003.

MILLER, J. E.; HOROHOV, D. W. Immunological aspects of nematode parasite control in sheep. Journal of Animal Science, Madison, v. 84, p. 124-132, 2006. Suplemento.

MOLENTO, M. B.; TASCA, C.; GALLO, A.; FERREIRA, M.; BONONI, R.; STECCA, E. Método Famacha como parâmetro clínico individual de infecção por Haemonchus contortus em pequenos ruminantes. Ciência Rural, Santa Maria, v. 34, n. 4, p. 1139-1145, 2004.

MORTENSEN, L. L.; WILLIAMSON, L. H.; TERRILL, T. H.; KIRCHER, R. A.; LARSEN, M.; KAPLAN, R. M. Evaluation of revalence and clinical implications of anthelmintic resistance in gastrointestinal nematodes of goats. Journal of the American Veterinary Medical Association, New York, v. 223, n. 4, p. 495-500, 2003.
NATIONAL RESEARCH COUNCIL - NRC. Nutrient requirements of domestic animals: nutrient requirements of sheep. Sixth Revised Edition, Washington, D.C.: National Academy Press, 1985. 99 p.

NELDER, J. A.; WEDDERBURN, W. M. Generalized linear models. Journal of the Royal Statistical Society, London, v. 135, n. 3, p. 370-84, 1972.

OTTO, C.; BONA, F. A.; SÁ, J. L.; FROEHNER, D. C.; BENATO, M. L. S.; SILVEIRA, M. Z. Eliminação de ovos de nematódeos por ovelhas naturalmente infectadas durante diferentes fases reprodutivas. Revista do Setor de Ciências Agrárias, Curitiba, v. 13, n. 1/2, p. 161-166, 1994.

PEGORARO, E. J.; POLI, C. H. E. C.; CARVALHO, P. C. F.; GOMES, M. J. T. M.; FISCHER, V. Manejo da pastagem de azevém, contaminação larval no pasto e infecção parasitária em ovinos. Pesquisa Agropecuária Brasileira, Brasília, v. 43, n. 10, p. 1397-1403, 2008.

ROCHA, R. A.; AMARANTE, A. F. T.; BRICARELLO, P. A. Resistance of Santa Inês and Ile de France suckling lambs to gastrointestinal nematode infections. Revista Brasileira de Parasitologia Veterinária, São Paulo, v. 14, n. 1, p. 17-20, 2005.

SECRETARIA DO ESTADO DO PARANÁ. Mapeamento dos municípios do Estado do Paraná. Curitiba: [s.n], 1985. 314 p.

STEAR, M. J.; BAIRDEN, K.; BISHOP, S. C.; BUITKAMP, J.; DUNCAN, J. L.; GETTINBY, G.; MCKELLAR, Q. A.; PARK, M,; PARKINS, J. J.; REID, S. W.; STRAIN, S.; MURRAY, M. The genetic basis of resistance to Ortestagia circumcincta in lambs. The Veterinary Journal, United Kingdom, v. 154, n. 2, p. 111119, 1997.

THOMAZ-SOCCOL, V.; SOUZA, F. P.; SOTOMAIOR, C.; CASTRO, E. A.; MILCZEWSKI, V.; MOCELIN, G.; SILVA, M. C. P. Resistance of Gastrointestinal Nematodes to Anthelmintics in Sheep (Ovis aries). Brazilian Archives of Biology and Technology, Curitiba, v. 47, n. 1, p. 41-47, 2004.

UENO, H.; GONÇALVES, P. C. Manual para diagnóstico das helmintoses de ruminantes. 3. ed. Tókio: Japan International Cooperation Agency, 1994. 166 p.

VIEIRA, L. S. O. Controle de verminose na produção orgânica de caprinos e ovinos. Disponível em: $<\mathrm{http}: / /$ www.cnpc.embrapa.br/verminose.htm >. Acesso em: 10 fev. 2010. 